\title{
Disorder Effects in BCS-BEC Crossover Region of Attractive Hubbard Model.
}

\author{
${ }^{a}$ E. Z. Kuchinskii ${ }^{1},{ }^{a}$ N. A. Kuleeva ${ }^{2)},{ }^{a, b}$ M. V. Sadovskii ${ }^{3)}$ \\ ${ }^{a}$ Institute for Electrophysics, Russian Academy of Sciences, Ural Branch, Amundsen str. 106, Ekaterinburg, 620016 \\ ${ }^{b}$ Institute for Metal Physics, Russian Academy of Sciences, Ural Branch, S. Kovalevskaya str. 18, Ekaterinburg, 620990
}

\begin{abstract}
We study the disorder effects upon superconducting transition temperature $T_{c}$ and the number of local pairs in attractive Hubbard model within the combined Nozieres - Schmitt-Rink and DMFT $+\Sigma$ approximations. We analyze the wide range of attractive interaction $U$, from the weak coupling region, where instability of the normal phase and superconductivity are well described by BCS model, to the limit of strong coupling, where superconducting transition is determined by Bose-Einstein condensation of compact Cooper pairs, forming at temperatures much higher than superconducting transition temperature. It is shown that disorder can either suppress $T_{c}$ in the weak coupling limit, or significantly enhance $T_{c}$ in the case of strong coupling. However, in all cases we actually prove the validity of generalized Anderson theorem, so that all changes of $T_{c}$ are related to change of the effective bandwidth due to disorder. Similarly, disorder effects on the number of local pairs are only due to these band-widening effects.
\end{abstract}

PACS: 71.10.Fd, 74.20.-z, 74.20.Mn

\section{INTRODUCTION}

The problem of superconductivity in the limit of strong coupling has attracted theorists for rather long time [1]. The significant progress in this field was 'achieved by Nozieres and Schmitt-Rink [2], who proposed an effective method to study the crossover from 'weak coupling BCS behavior to Bose-Einstein conden'sation (BEC) in strong coupling region. In recent years the progress of experimental studies of ultracold quantum gases in magnetic and optical dipole traps, as well as in optical lattices, allowing controllable change of density and interaction parameters (see reviews $[3,4]$ ) has also increased the interest to studies of BCS-BEC crossover. One of the simplest models allowing the 'study of BCS-BEC crossover is the Hubbard model with attractive interaction.

The most effective theoretical method to study 'strongly correlated systems both in the case of repulsive interactions and in the case of attraction (including the region of BCS-BEC crossover) is the dynamical meanfield theory (DMFT) $[5,6,7]$. Within the framework of DMFT the attractive Hubbard model has already been studied in the number of papers $[8,9,10,11]$. However, there are only few works devoted to the studies of

\footnotetext{
3) E-mail: kuchinsk@iep.uran.ru

3) E-mail: strigina@iep.uran.ru

3) E-mail: sadovski@iep.uran.ru
}

disorder effects on the properties of normal and superconducting phases in this model. Qualitatively the influence of disorder on the superconducting critical temperature $T_{c}$ in the region of BCS-BEC crossover was studied in Ref. [12]. Diagrammatic approach to the analysis of disorder effects upon $T_{c}$ and normal phase properties in the crossover region was developed in Ref. [13]. Recently we have studied [14] the disorder influence on single-particle properties and optical conductivity in disordered attractive Hubbard model within our general $\mathrm{DMFT}+\Sigma$ approach [15], which is especially convenient to take into account different additional interactions like scattering by short-range order parameter fluctuations $[16,17,18,19]$, disorder $[20,21]$ or electron-phonon interaction [22]. In this paper we use DMFT $+\Sigma$ approach combined with Nozieres - Schmitt-Rink approximation

[2] to study the influence of disorder upon superconducting transition temperature $T_{c}$ and the number of local pairs in attractive Hubbard model for the wide range of interaction parameter $U$, including the BCSBEC crossover region.

\section{BASICS OF NOZIERES - SCHMITT-RINK AND DMFT $+\Sigma$ APPROACHES.}

We shall consider disordered attractive Hubbard model with the Hamiltonian:

$$
H=-t \sum_{\langle i j\rangle \sigma} a_{i \sigma}^{\dagger} a_{j \sigma}+\sum_{i \sigma} \epsilon_{i} n_{i \sigma}-U \sum_{i} n_{i \uparrow} n_{i \downarrow}
$$


where $t>0$ is the transfer integral between nearest neighbors on the lattice, $U$ is Hubbard onsite attraction, $n_{i \sigma}=a_{i \sigma}^{\dagger} a_{i \sigma}$ is electron number operator on the lattice site, $a_{i \sigma}\left(a_{i \sigma}^{\dagger}\right)$ is electron annihilation (creation) operator with spin projection $\sigma$ and local energies $\epsilon_{i}$ are assumed to be independent random variables on different lattice cites. To simplify diagrammatic analysis we assume the Gaussian distribution for $\epsilon_{i}$ :

$$
\mathcal{P}\left(\epsilon_{i}\right)=\frac{1}{\sqrt{2 \pi} \Delta} \exp \left(-\frac{\epsilon_{i}^{2}}{2 \Delta^{2}}\right)
$$

Parameter $\Delta$ here is the measure of disorder and the Gaussian random field with short-ranged ("whitenoise") correlations is equivalent to the usual "impurity" scattering, leading the the standard diagram technique for the averaged Green's functions [23].

In the following we shall consider the model system with "bare" semi-elliptic density of states (per elementary lattice cell and one spin projection) given by:

$$
N_{0}(\varepsilon)=\frac{2}{\pi D^{2}} \sqrt{D^{2}-\varepsilon^{2}}
$$

so that the bandwidth is $W=2 D$. All calculations below were made for the case of quarter-filled band (electron density per cite $\mathrm{n}=0.5)$.

In the absence of disorder superconducting transition temperature was analyzed in this model in a number of papers $[8,9,11]$ both from the condition of Cooper instability of the normal phase [8] (divergence of Cooper susceptibility) and also from the condition of superconducting order parameter becoming zero at $T_{c}[9,11]$. In Ref. [14] we have determined this critical temperature from the condition of instability of the normal phase, as reflected in specific instability of DMFT iteration procedure. The results obtained in this way in fact just coincide with the results of Refs. [8, 9, 11].

The essence of Nozieres - Schmitt-Rink approach [2] to calculation of $T_{c}$ in the wide region of coupling strengths $U$, providing an effective interpolation from weak to strong coupling (including the BCS-BEC crossover region) is to solve the BCS equation for transition temperature:

$$
1=\frac{|U|}{2} \int_{-\infty}^{\infty} d \varepsilon N_{0}(\varepsilon) \frac{t h \frac{\varepsilon-\mu}{2 T_{c}}}{\varepsilon-\mu},
$$

jointly with an equation for chemical potential (implicitly determined by the band-filling), which actually controls $T_{c}$ in strong coupling BEC region. In Ref. [14] we have shown that such calculations, with an equation for chemical potential solved via DMFT, produce the dependence $T_{c}$ on $U$, which is in almost quantitative agreement with results obtained via much more timeconsuming exact DMFT calculations. This is rather surprising, because of neglect of all vertex corrections due to $U$ (ladder approximation) in Eq. (4), especially in the region of large $U$. Apparently this signifies rather small role of these vertex corrections (fluctuation effects) for BCS-like instability both in crossover and strong coupling regions. However, in calculations of chemical potential $\mu$ (controlling $T_{c}$ for large $U$ ) these corrections are quite important and only their correct account within DMFT allows us to obtain the correct behavior of $T_{c}$ in the limit of large $U$.

This allows us to calculate $T_{c}$ for the case of disordered attractive Hubbard model using the same approach. Actually, we shall solve Eq. (4), from which all corrections due to disorder scattering just drop out, except those leading to disorder widening of the density of states [24] (replacing $N_{0}(\varepsilon)$ in Eq. (4) by disorder renormalized density of states), jointly with an equation for chemical potential, obtained via $\mathrm{DMFT}+\Sigma$ procedure [15], which takes into contributions due to disorder, producing the the chemical potential for different values of $U$ and disorder $\Delta$.

This generalized DMFT $+\Sigma$ approach $[16,17,18$, 15] supplies the standard dynamical mean-field theory (DMFT) $[5,6,7]$ with an additional ("external") selfenergy $\Sigma_{\mathbf{p}}(\varepsilon)$ (which can in general be momentum dependent), taking into account any possible interaction outside the DMFT, which gives an effective calculation method for either single-particle or two-particle properties $[19,20]$. The success of this generalized approach is connected with the choice of the single-particle Green's function in the following form:

$$
G(\varepsilon, \mathbf{p})=\frac{1}{\varepsilon+\mu-\varepsilon(\mathbf{p})-\Sigma(\varepsilon)-\Sigma_{\mathbf{p}}(\varepsilon)},
$$

where $\varepsilon(\mathbf{p})$ is the "bare" electronic dispersion, while the total self-energy is given by the additive sum of local $\Sigma(\varepsilon)$, determined by DMFT, and "external" $\Sigma_{\mathbf{p}}(\varepsilon)$, thus neglecting any interference between Hubbard and "external" interactions. This allows us to preserve the structure of self-consistent equations of the standard DMFT $[5,6,7]$. Hovewer, there are two major difference with traditional DMFT. During each DMFT iteration step we recalculate an "external" self-energy $\Sigma_{\mathbf{p}}(\varepsilon)$ using some approximate scheme, taking into account additional interactions, and the local Green's function is "dressed" by $\Sigma_{\mathbf{p}}(\varepsilon)$ at each iteration step.

Below for an "external" self-energy due to disorder scattering, entering DMFT $+\Sigma$ cycle, we use the simplest approximation neglecting "crossing" diagrams, i.e. the self-consistent Born approximation, which in case of 
Gaussian distribution of site energies takes the (momentum independent) form:

$$
\Sigma_{\mathbf{p}}(\varepsilon) \rightarrow \tilde{\Sigma}(\varepsilon)=\Delta^{2} \sum_{\mathbf{p}} G(\varepsilon, \mathbf{p})
$$

where $G(\varepsilon, \mathbf{p})$ is the single-electron Green's function (5) and $\Delta$ is the disorder amplitude.

To solve the effective Anderson impurity problem of DMFT below we use the numerical renormalization group approach [25].

\section{MAIN RESULTS.}

In Fig. 1 we show the dependence of superconducting transition temperature, normalized by the critical temperature in the absence of disorder $\left(T_{c 0}=T_{c}(\Delta=0)\right)$, for quarter-filled band $(n=0.5)$ for different values of attractive interaction $U$. We can see that in the case of weak coupling $(U / 2 D \ll 1)$ disorder somehow suppresses $T_{c}$ (curve 1). At intermediate couplings $(U / 2 D \sim 1)$ weak disorder leads to the growth of $T_{c}$, while the further increase of disorder suppresses the critical temperature (curves 2 and 3). In the strong coupling region $(U / 2 D \gg 1)$ the growth of disorder leads to significant increase of the critical temperature (curves 4 and 5).

However, this complicated dependence of superconducting critical temperature on disorder is easily explained by the conduction band widening by growing disorder. In Fig. 2 the black curve with pentagonal data points represents the dependence of critical temperature

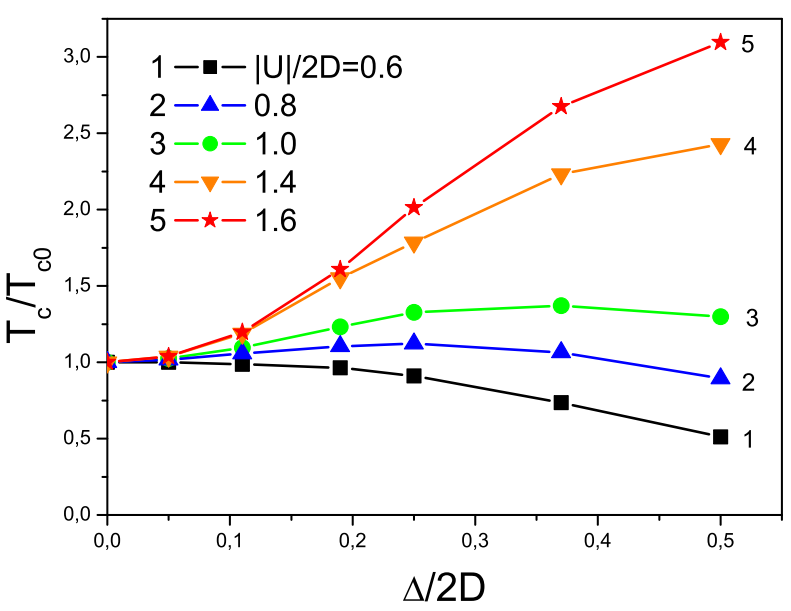

Fig. 1. Dependence of superconducting critical temperature on disorder for different values of Hubbard attraction.

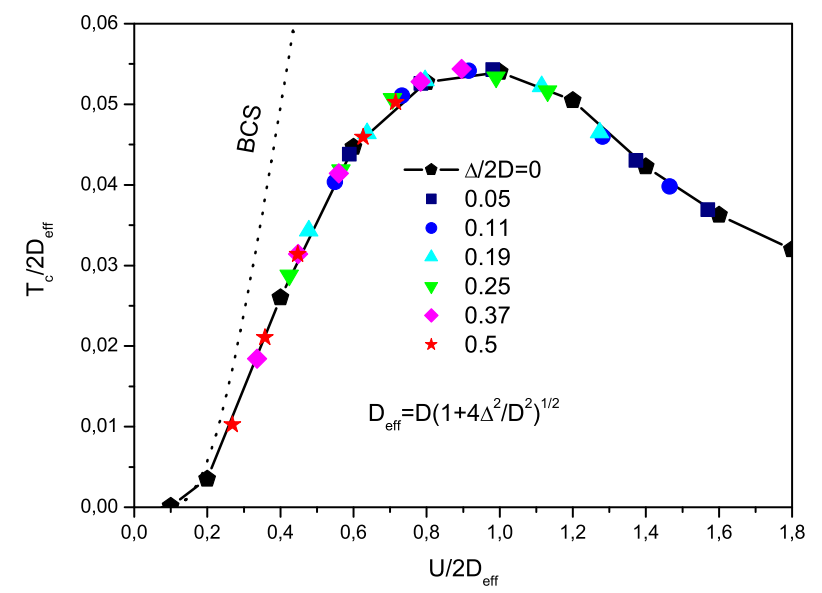

Fig. 2. Universal dependence of superconducting critical temperature on the strength of Hubbard attraction for different values of disorder.

$T_{c} / 2 D$ on attraction strength $U / 2 D$ in the absence of disorder $(\Delta=0)$ in Nozieres - Schmitt-Rink approximation [14]. The growth of disorder leads to the effective widening of the conduction band, so that in in our selfconsistent Born approximation for disorder scattering (6) the semi-elliptic form of the density of states does not change, while the effective half-bandwidth grows as [20]:

$$
D_{e f f}=D \sqrt{1+4 \frac{\Delta^{2}}{D^{2}}}
$$

The other data points shown in Fig. 2 represent the results of our calculations in the combined Nozieres Schmitt-Rink and DMFT $+\Sigma$ approximations for different values of disorder. We can see that all data points as expressed via appropriately scaled variables $U / 2 D_{\text {eff }}$ and $T_{c} / 2 D_{\text {eff }}$ perfectly follow the universal curve, obtained in the absence of disorder. These results illustrate, at least in approximations used here, the validity of the generalized Anderson theorem [24, 26] (for all couplings, including the BCS-BEC crossover and strong coupling regions) - the critical temperature of superconducting transition (for the case of $s$-wave pairing) is affected by disorder only through the appropriate change of electron bandwidth (density of states). From Fig. 2 we can see, that in the weak coupling region $U / 2 D_{\text {eff }} \ll 1$ the critical temperature in this approximation is close to that obtained in the usual BCS model (dashed curve in Fig. 2). For $U / 2 D_{\text {eff }} \sim 1$ the critical temperature $T_{c}$ reaches the maximum. For $U / 2 D_{\text {eff }} \gg 1$ it drops with the growth of $U$, showing $T_{c} \sim 1 / U$ behavior [2], as in the strong coupling region $T_{c}$ is determined by the condition of Bose-Einstein con- 


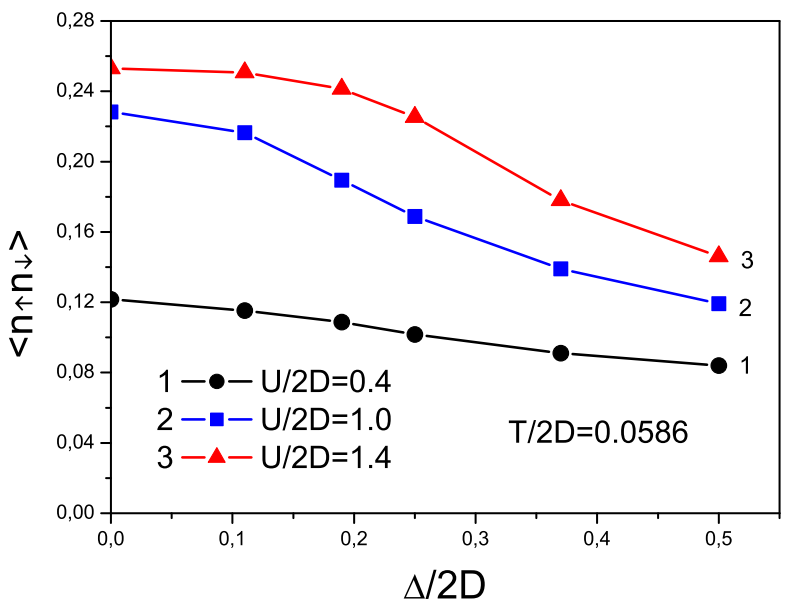

Fig. 3. Dependence of the number of local pairs on disorder for different values of Hubbard attraction.

densation of Cooper pairs and hopping motion of these pairs (via virtual ionization) appears only in the second order of perturbation theory being proportional to $t^{2} / U$ $[2]$.

Band widening due to disorder also leads to the effective suppression of the number of local pairs (doubly occupied sites). The average number of local pairs is determined by pair correlation function $\left\langle n_{\uparrow} n_{\downarrow}\right\rangle$, which in the absence of disorder grows with the increase of Hubbard attraction $U$ from $<n_{\uparrow} n_{\downarrow}>=<n_{\uparrow}><$ $n_{\uparrow}>=n^{2} / 4$ for $U / 2 D_{\text {eff }} \ll 1$ to $<n_{\uparrow} n_{\downarrow}>=n / 2$ for $U / 2 D_{\text {eff }} \gg 1$, when all electrons are paired. The growth of $D_{\text {eff }}$ with disorder leads to an effective suppression of the parameter $U / 2 D_{\text {eff }}$ and corresponding suppression of the number of doubly occupied sites. In Fig. 3 we show the disorder dependence of the number of doubly occupied sites for three different values of Hubbard attraction. We see that in all cases the growth of disorder suppresses the number of doubly occupied sites (local pairs). In fact, similarly to $T_{c}$, the change of the number of local pairs with disorder can be attributed only to the change of the effective bandwidth of the "bare" band (7) with the growth of disorder. In Fig. 4 the curve with black squares shows the dependence of the number of doubly occupied sites on Hubbard attraction for the case of quarter-filled band $(n=0.5)$ in the absence of disorder at temperature $T / 2 D=0.0586$. This curve is actually universal the dependence of the number of local pairs $\left\langle n_{\uparrow} n_{\downarrow}\right\rangle$ on the scaled parameter $U / 2 D_{\text {eff }}$ with appropriately scaled temperature $T / 2 D_{\text {eff }}=0.0586$ in the presence of disorder is given by the same curve, which as shown by by circles, representing data obtained for five differ-

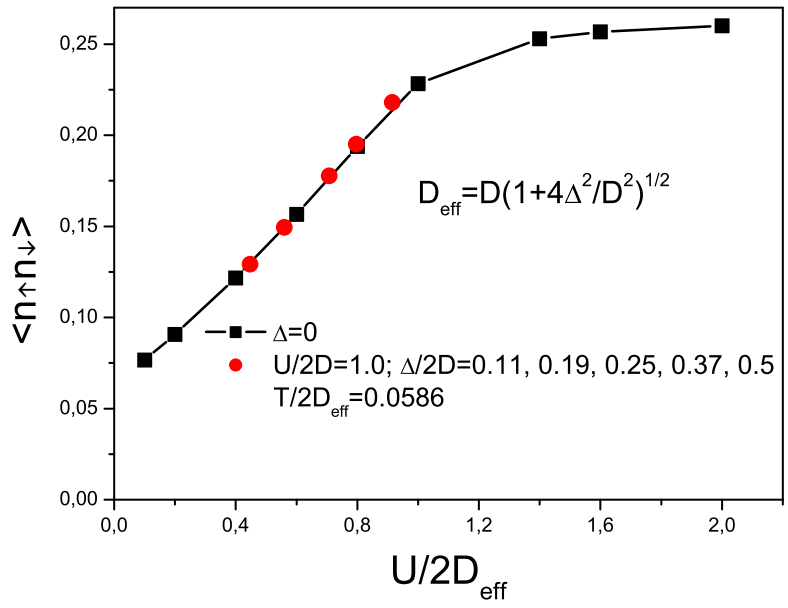

Fig. 4. Universal dependence of the number of local pairs on the the strength of Hubbard attraction for different values of disorder.

ent disorder levels and shown in Fig. 4 for the case of $U / 2 D=1$.

\section{CONCLUSION.}

In this paper, using the combined Nozieres Schmitt-Rink and DMFT $+\Sigma$ approximations we have investigated the influence of disorder on superconducting critical temperature and the number of local pairs in disordered attractive Hubbard model. We have studied the wide range of attractive couplings $U$, from the weak coupling region of $U / 2 D_{\text {eff }} \ll 1$, where normal phase instability and superconductivity is described by BCS model, to the strong coupling region of $U / 2 D_{\text {eff }} \gg 1$, where superconducting transition is related to Bose-Einstein condensation of preformed Cooper pairs, which appear in the system at temperatures significantly higher, than superconducting transition temperature. Disorder can either suppress the critical temperature $T_{c}$ in the case of weak coupling, or significantly increase $T_{c}$ in the of strong coupling. However, these dependences in fact confirm the validity of the generalized Anderson theorem - all changes of superconducting critical temperature can be attributed to general widening of conduction band by disorder (for the case of $s$-wave pairing, which can only be realized in the attractive Hubbard model). In the weak coupling region transition temperature is well described by BCS model, while in the strong coupling region it is determined by the condition of Bose-Einstein condensation and drops with the growth of $|U|$ as $1 /|U|$, passing the maximum at $|U| / 2 D_{\text {eff }} \sim 1$. Similarly, only the band 
widening by disorder is responsible for the change of the number of local pairs (doubly occupied sites). The growth of disorder leads to the effective drop of the ratio $U / 2 D_{\text {eff }}$ and corresponding drop of the number of local pairs.

This work is supported by RSF grant No. 14-1200502 .

1. A. J. Leggett, in Modern Trends in the Theory of Condensed Matter, edited by A. Pekalski and J. Przystawa (Springer, Berlin 1980).

2. P. Nozieres and S. Schmitt-Rink, J. Low Temp. Phys. 59, 195 (1985)

3. I. Bloch, J. Dalibard, and W. Zwerger. Rev. Mod. Phys. 80, 885 (2008)

4. L.P. Pitaevskii. Usp. Fiz. Nauk, 176, No. 4, 345 (2006)

5. Th. Pruschke, M. Jarrell, and J. K. Freericks, Adv. in Phys. 44, 187 (1995).

6. A. Georges, G. Kotliar, W. Krauth, and M. J. Rozenberg, Rev. Mod. Phys. 68, 13 (1996).

7. D. Vollhardt in "Lectures on the Physics of Strongly Correlated Systems XIV", eds. A. Avella and F. Mancini, AIP Conference Proceedings vol. 1297 (American Institute of Physics, Melville, New York, 2010), p. 339; ArXiV: 1004.5069

8. M. Keller, W. Metzner, and U. Schollwock. Phys. Rev. Lett. 86, 46124615 (2001); ArXiv: cond-mat/0101047

9. A. Toschi, P. Barone, M. Capone, and C. Castellani. New Journal of Physics 7, 7 (2005); ArXiv: condmat/0411637v1

10. J. Bauer, A.C. Hewson, and N. Dupis. Phys. Rev. B 79, 214518 (2009); ArXiv: 0901.1760v2

11. A. Koga and P. Werner. Phys. Rev. A 84, 023638 (2011); ArXiv: 1106.4559v1

12. A.I. Posazhennikova and M.V. Sadovskii. Pisma Zh. Eksp. Teor. Fiz. 65, 258 (1997) [JETP Letters 65, 270 (1997)]

13. F. Palestini, G.C. Strinati. ArXiv:1311.2761

14. N.A. Kuleeva, E.Z. Kuchinskii, M.V. Sadovskii. Zh. Eksp. Teor. Fiz. 146, No. 1 (2014) [JETP 119, No. 1 (2014)](in press); ArXiv: 1401.2295

15. E.Z. Kuchinskii, I.A. Nekrasov, M.V. Sadovskii. Usp. Fiz. Nauk 182, No. 4, 345-378 (2012) [Physics - Uspekhi 55, No. 4, 325-255 (2012)]; ArXiv:1109.2305

16. E.Z.Kuchinskii, I.A.Nekrasov, M.V.Sadovskii. Pisma Zh. Eksp. Teor. Fiz. 82, No. 4, 217 (2005) [JETP Lett. 82, 198 (2005)]; ArXiv: cond-mat/0506215.

17. M.V. Sadovskii, I.A. Nekrasov, E.Z. Kuchinskii, Th. Prushke, V.I. Anisimov. Phys. Rev. B 72, No 15, 155105 (2005); ArXiV: cond-mat/0508585

18. E.Z. Kuchinskii, I.A. Nekrasov, M.V. Sadovskii. Fizika Nizkikh Temperatur 32, 528-537 (2006) [Low Temp. Phys. 32, 398 (2006)]; ArXiv: cond-mat/0510376
19. E.Z. Kuchinskii, I.A. Nekrasov, M.V. Sadovskii. Phys. Rev. B 75, 115102-115112 (2007); ArXiv: condmat/0609404.

20. E.Z. Kuchinskii, I.A. Nekrasov, M.V. Sadovskii, Zh. Eksp. Teor. Fiz. 133, No.3 , 670 (2008) [JETP 106, No. 3, 581 (2008)]; ArXiv: 0706.2618.

21. E.Z.Kuchinskii, N.A.Kuleeva, I.A.Nekrasov, M.V.Sadovskii. Zh. Eksp. Teor. Fiz. 137, No 2, 368 (2010) [JETP 110, No. 2, 325-335 (2010)]; ArXiv: 0908.3747

22. E.Z.Kuchinskii, I.A.Nekrasov, M.V.Sadovskii. Phys. Rev. B 80, 115124 (2009); ArXiv: 0906.3865

23. A.A. Abrikosov, L.P. Gorkov, I.E. Dzyaloshinskii. Quantum Field TheoreticalMethods in Statistical Physics. Pergamon Press, Oxford 1965; M. V. Sadovskii. Diagrammatics. World Scientific, Singapore 2006

24. M.V. Sadovskii. Superconductivity and Localization. World Scientific, Singapore 2000

25. R. Bulla, T.A. Costi, T. Pruschke, Rev. Mod. Phys. 60, 395 (2008).

26. P.G. De Gennes. Superconductivity of Metals and Alloys. W.A. Benjamin, NY 1966 